\title{
All-trans retinoic acid inhibits migration, invasion and proliferation, and promotes apoptosis in glioma cells in vitro
}

\author{
CHEN LIANG $^{1}$, LING YANG ${ }^{2}$ and SHIWEN GUO ${ }^{1}$ \\ ${ }^{1}$ Department of Neurosurgery, First Affiliated Hospital of Xi'an Jiaotong University; \\ ${ }^{2}$ Department of Aeromedical Physical Examination, Xi'an Civil Aviation Hospital, Xi'an, \\ Shaanxi 710061, P.R. China
}

Received May 27, 2014; Accepted March 17, 2015

DOI: $10.3892 / \mathrm{ol} .2015 .3120$

\begin{abstract}
All-trans retinoic acid (ATRA) is a derivative of vitamin A that can induce differentiation and apoptosis, as well as inhibit proliferation, in glioma cells. However, the effect of ATRA on the migration and invasiveness of glioma remains poorly understood. In addition, although it is universally accepted that ATRA can induce apoptosis and inhibit proliferation in glioma cells, the association between the concentration and effects of ATRA remain unclear. Therefore, the present study investigated the effects of ATRA treatment on the migration, invasion, apoptosis and proliferation of glioma cells. The U87 and SHG44 glioma cell lines were treated with various concentrations of ATRA, consisting of $0,5,10,20$ and $40 \mu \mathrm{mol} / 1$. A scratch wound healing assay and a Matrigel invasion assay were used to investigate cell migration and invasion, respectively. Flow cytometry was performed to investigate apoptosis and cell cycle distribution. Reverse transcription-quantitative polymerase chain reaction and western blotting were used to investigate the expression of matrix metalloproteinase (MMP)-2 and -9 in each cell treatment group. Following treatment with ATRA, the migration, invasion and proliferation of the glioma cells were significantly inhibited, and the apoptosis rate was significantly increased compared with that of the blank control group. Furthermore, a dose-effect association was identified between each effects and ATRA treatment. The mRNA and protein expression of MMP-2 in U87 glioma cells was not significantly affected following treatment with low concentrations of ATRA, consisting of 5 and $10 \mu \mathrm{mol} / \mathrm{l}$ ATRA, compared with the expression in the control group $(\mathrm{P}>0.05)$. However, treatment with high concentrations of ATRA, consisting of 20 and $40 \mu \mathrm{mol} / \mathrm{l}$ ATRA, significantly downregulated the expression
\end{abstract}

Correspondence to: Professor Shiwen Guo, Department of Neurosurgery, First Affiliated Hospital of Xi'an Jiaotong University, 277 Yanta West Road, Xi'an, Shaanxi 710061, P.R. China

E-mail: guoshiwen1962@126.com

Key words: glioma, proliferation, all-trans retinoic acid, migration, invasion levels of MMP-2 in U87 cells. In contrast to U87 cells, the administration of ATRA treatment to SHG44 glioma cells resulted in a significant and dose-dependent downregulation in MMP-2 mRNA and protein expression $(\mathrm{P}<0.01)$. In addition, significant downregulation of MMP-9 expression was identified in the two glioma cell lines $(\mathrm{P}<0.01)$. The results of the present study indicate that treatment with ATRA may inhibit migration, invasion and proliferation, and promote apoptosis in glioma cells. Furthermore, the current study indicates that the inhibition of glioma cell invasion by ATRA may be partially associated with its effect ability to downregulate MMP expression.

\section{Introduction}

Worldwide, glioma is one of the most frequent types of brain tumor in adults. The current standard therapeutic regime includes maximal safe surgical resection combined with radiotherapy and temozolomide chemotherapy (1). Due to the development of such treatment strategies, the percentage of patients alive two years after diagnosis has increased to $26 \%$ (2). However, the prognosis of patients with glioma remains unsatisfactory. For example, the median survival time of patients with glioblastoma multiforme is only 14.6 months subsequent to standard therapy (3). Therefore, the identification of more effective treatment strategies for patients with glioma is required.

All-trans retinoic acid (ATRA) is a derivative of vitamin A that exerts its effects through retinoic acid receptors (RARs) and retinoic $\mathrm{X}$ receptors. Various studies have demonstrated that ATRA can induce differentiation and apoptosis $(4,5)$, as well as induce cell growth arrest, in glioma cells (6). Furthermore, a previous study revealed that ATRA may enhance the bystander effect of suicide-gene therapy against medulloblastoma (7), with a number of studies demonstrating that treatment with ATRA in combination with specific chemoimmunotherapeutic agents may significantly enhance its antitumor effect on glioma (8-12). These results indicate the therapeutic potential of ATRA for patients with glioma.

The invasion of glioma is crucial to tumor progression. Malignant glioma, such as glioblastoma, can rapidly invade into neighboring brain structures and spread through infiltration. This invasion is largely dependent on matrix metalloproteinases (MMPs). MMPs, particularly MMP-2 and 
MMP-9, are known to be major glioma invasion-mediating factors that degrade the extracellular matrix to create space for invading glioma cells (13). However, the effect of ATRA on the migration and invasion of glioma cells remains poorly understood. In addition, although it is universally accepted that ATRA can induce the apoptosis and inhibit the proliferation of glioma cells, the association between the concentration and effects of ATRA remain unclear. Therefore, the present study aimed to investigate the effects of ATRA treatment on the migration, invasion, apoptosis and proliferation of glioma cells, with the intention of partially revealing the anti-glioma mechanisms of ATRA.

\section{Materials and methods}

Materials. U-87MG and SHG44 human glioma cell lines were purchased from the Cell Resource Center of the Chinese Academy of Sciences (Shanghai, China).

Cell culture. U87 cells were cultured in DMEM (Hyclone Laboratories, Inc., Beijing, China) supplemented with $10 \%$ fetal bovine serum (FBS; Hyclone Laboratories, Inc.) in an atmosphere of $5 \% \mathrm{CO}_{2}$ at $37^{\circ} \mathrm{C}$. The SHG44 cells were cultured in RPMI 1640 (Hyclone Laboratories, Inc.) supplemented with $10 \% \mathrm{FBS}$ in a $5 \% \mathrm{CO}_{2}$ atmosphere at $37^{\circ} \mathrm{C}$.

Scratch woundhealing assay. The U87MG and SHG44 cells were seeded at a density of $1 \times 10^{5}$ cells/well in 24-well plates (Corning Life Sciences, Lowell, MA, USA). After $24 \mathrm{~h}$, the cell monolayer was scraped in a straight line using a $20-\mu 1$ pipette tip and the cells were washed three times with phosphate-buffered saline. ATRA (Sigma-Aldrich, St. Louis, MO, USA) was dissolved in dimethyl sulfoxide (DMSO; Sigma-Aldrich) and stored in light-protected vials at $-20^{\circ} \mathrm{C}$ as stock solution. The stock solution was diluted to the desired concentrations immediately prior to use and all experiments were performed under low-light conditions to minimize ATRA photoisomerization. The cells were incubated in low-serum media containing $0.5 \% \mathrm{FBS}$ and various concentrations of ATRA $(5,10,20$ and $40 \mu \mathrm{mol} / \mathrm{l})$ and the control group was treated with an equal volume of solvent (DMSO) in culture medium. All the plates were placed in a culture incubator at $37^{\circ} \mathrm{C}$ and at $0,6,12$ and $24 \mathrm{~h}$ after incubation, the plates were removed from the incubator for images to be captured under a phase-contrast microscope (CKX41; Olympus Corporation, Tokyo, Japan). Image-Pro Plus software (Media Cybernetics, Inc., Rockville, MD, USA) was used to measure the relative migration distances.

Matrigel invasion assay. The U87MG and SHG44 cells were plated at a density of $5 \times 10^{5}$ cells/well on Transwell chambers (Corning Life Sciences) precoated with $50 \mu 1$ Matrigel (BD Biosciences, Bedford, MA, USA) diluted with culture medium. Serum-free culture medium containing 5, 10,20 or $40 \mu \mathrm{mol} / 1$ ATRA was used to incubate the cells of each group and medium containing 20\% FBS in the lower chamber served as the chemoattractant. Non-invading cells were removed with cotton swabs after $24 \mathrm{~h}$, while invading cells were dyed with $0.1 \%$ crystal violet (Amresco, Inc., Solon, $\mathrm{OH}, \mathrm{USA}$ ) and counted using Image-Pro Plus software under a phase-contrast microscope (magnification, x200). The average cell number in five random visual fields was considered to be the number of invading cells of each chamber.

Flow cytometry. The U87MG and SHG44 cells were seeded at a density of $3 \times 10^{5}$ cells/well in six-well plates (Corning Life Sciences) with 5, 10, 20 or $40 \mu \mathrm{mol} / 1$ ATRA for $24 \mathrm{~h}$. An Annexin V-fluorescein isothiocyanate cell apoptosis detection kit (Beyotime Institute of Biotechnology, Haimen, Jiangsu, China) was used to detect cell apoptosis and a cell cycle analysis kit (Beyotime Institute of Biotechnology) was used to detect the cell cycle position of each group, according to the manufacturers' instructions. Furthermore, the proliferation index (PI) was used to indicate the proliferation level of each group, according to the following equation: $\mathrm{PI}=(\mathrm{S}+\mathrm{G} 2 / \mathrm{M}) /(\mathrm{G} 0 / \mathrm{G} 1+\mathrm{S}+\mathrm{G} 2 / \mathrm{M}) \times 100 \%$.

Quantitative polymerase chain reaction ( $q P C R)$. The U87MG and SHG44 cells were seeded at a density of 3x10 $10^{5}$ cells/well in six-well plates with various concentrations of ATRA for $24 \mathrm{~h}$, as aforementioned. The cells were then lysed and the total RNA was isolated using RNA Fast 200 kit (Shanghai Fastagen Biotechnology Co., Ltd., Shanghai, China), according to the manufacturer's instructions. RNA was reverse-transcribed using a PrimeScript RT Master Mix kit (Takara Biotechnology Co., Ltd., Dalian, China). qPCR was performed using SYBR Premix Ex Taq II (Takara Biotechnology Co., Ltd.) on an iQ5 thermal cycler and analyzed using iQ5 software, version 2.0 (Bio-Rad, Hercules, CA, USA). Gene expression was compared using the cycle threshold $(\mathrm{Ct})$. $\mathrm{Ct}$ was defined using the following equation: $\Delta \mathrm{Ct}=\mathrm{Ct}_{\text {Target }}-\mathrm{Ct}_{\beta \text {-actin }}$, where $\beta$-actin expression was used as the endogenous reference gene. Change in gene expression was evaluated using the $2^{-\Delta \Delta \mathrm{Ct}}$ method (14). All primers were designed and synthesized by Takara Biotechnology Co., Ltd. (Table I).

Western blotting. The U87MG and SHG44 cells were cultured in $25 \mathrm{ml}$ cell culture flasks (Corning Life Sciences) with various concentration of ATRA for $24 \mathrm{~h}$. The cells were then harvested for use in assays. The cells were washed twice with PBS and then scraped in $300 \mu$ l radioimmunoprecipitation assay lysis buffer (Beyotime Institute of Biotechnology) with $1 \mathrm{mmol} / 1$ phenylmethanesulfonyl fluoride on ice. The lysates were cleared of insoluble material by centrifugation, the protein concentration was determined using a Bradford protein assay kit (Beyotime Institute of Biotechnology) and samples were boiled in 1X SDS-PAGE sample loading buffer, resolved by SDS-PAGE, and then transferred to nitrocellulose membranes (Bio-Rad Laboratories). The membranes were probed with rabbit anti-human MMP-2 (dilution, 1:500; cat. no. BS1236), rabbit anti-human MMP-9 (dilution, 1:500; cat. no. BS1241) and rabbit anti-human GAPDH (dilution, 1:5000; cat. no. ap0063) antibodies, all purchased from Bioworld Technology, Inc. (St. Louis Park, MN, USA). Subsequent to washing in Tris-buffered saline containing $0.02 \%$ Tween 20 (Sigma-Aldrich), the membranes were incubated with a secondary polyclonal anti-rabbit IgG antibody conjugated to horseradish peroxidase (dilution, 1:2000; Thermo Fisher Scientific, Pittsburgh, PA, USA). Membranes were developed in Supersignal West Pico chemiluminescent reagent (Pierce Biotechnology, Inc., Rockford, IL, USA). 

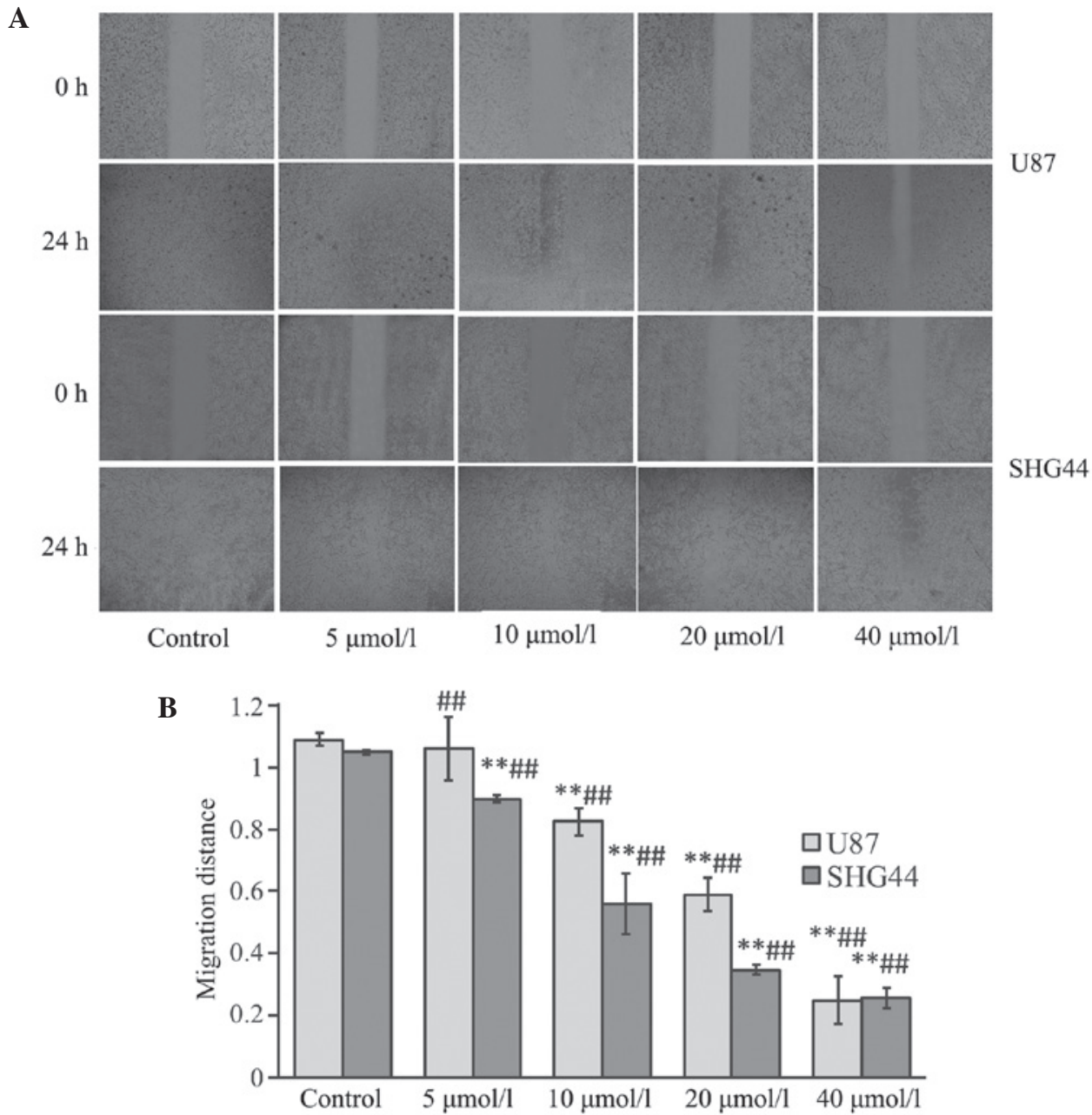

Figure 1. (A) Effect of all-trans retinoic acid (ATRA) on the migration of U87 and SHG44 glioma cells (magnification, x100). (B) Migration distance (mm) of glioma cells following treatment with various concentration of ATRA for $24 \mathrm{~h}$, indicating a significantly reduced migration distance in all treated cells, excluding U87 glioma cells treated with $5 \mu \mathrm{mol} / 1$ ATRA. Furthermore, the migration distance decreased with the increase in ATRA concentration. ${ }^{* *} \mathrm{P}<0.01$ vs. control group; ${ }^{\# \#} \mathrm{P}<0.01$ vs. any other treatment group $(\mathrm{n}=3)$.

Statistical analysis. The data are presented as the mean \pm standard deviation and were analyzed using SPSS software, version 17.0 (SPSS, Inc., Chicago, IL, USA). One-way analysis of variance was used to compare groups and Fisher's least significant difference tests were performed for subsequent comparisons between groups. $\mathrm{P}<0.05$ was considered to indicate a statistically significant difference.

\section{Results}

ATRA significantly inhibits the migration of glioma cells. Subsequent to treatment with various concentrations of ATRA for $24 \mathrm{~h}$, the migration distance of glioma cells was significantly reduced compared with the control group $(\mathrm{P}<0.01)$, excluding U87 glioma cells treated with $5 \mu \mathrm{mol} / 1$ ATRA $(\mathrm{P}>0.05)$. Furthermore, the migration distance significantly decreased with each increase in ATRA concentration $(\mathrm{P}<0.01$; Fig. 1).

ATRA significantly inhibits the invasion of glioma cells. Following treatment with different concentrations of ATRA for $24 \mathrm{~h}$, the number of invading cells was significantly reduced compared with that of the control group, particularly after treatment with high concentrations of ATRA, consisting of 20 and $40 \mu \mathrm{mol} / 1$ ATRA. A statistically significant difference was identified between all groups $(\mathrm{P}<0.01$; Fig. 2).

ATRA significantly inhibits proliferation and promotes apoptosis in glioma cells. Following treatment with various concentrations of ATRA for $24 \mathrm{~h}$, the PI of glioma cells in each treatment group was significantly decreased $(\mathrm{P}<0.01)$, particularly subsequent to treatment with 20 and $40 \mu \mathrm{mol} / \mathrm{l}$ ATRA (Fig. 3A).

Following treatment with various concentrations of ATRA for $24 \mathrm{~h}$, the apoptosis rate of glioma cells in each treatment group was significantly increased compared with the control group $(\mathrm{P}<0.01)$. Furthermore, a statistically significant difference was identified between each treatment group and all other treatment groups $(\mathrm{P}<0.01$; Fig. 3B).

ATRA significantly inhibits the expression of MMP-2 and $M M P-9$. Following a 24-h incubation with the aforementioned concentrations of ATRA, various effects occurred on the MMP-2 mRNA expression level in glioma cells, as indicated in Fig. 4A. Lower concentrations of ATRA (5 and $10 \mu \mathrm{mol} / \mathrm{l}) \mathrm{did}$ not influence the expression of MMP-2 mRNA in U87 glioma 
A U87
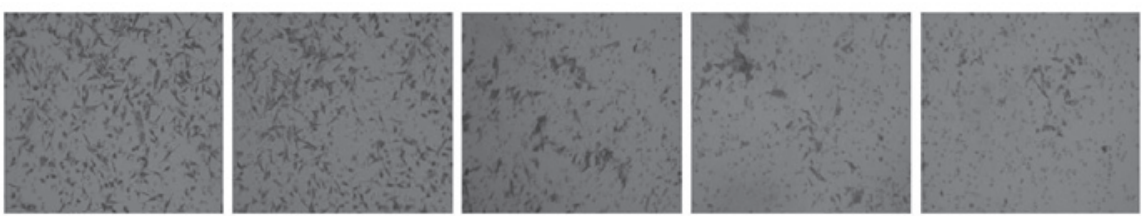

SHG44

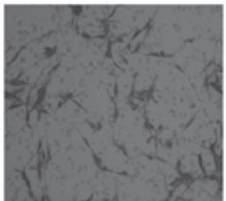

Control

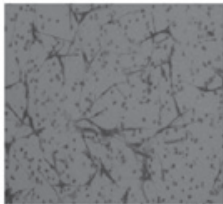

$5 \mu \mathrm{mol} / 1$

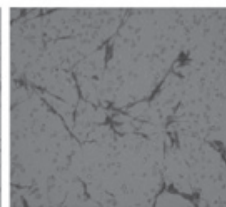

$10 \mu \mathrm{mol} / \mathrm{l}$

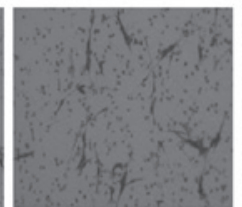

$20 \mu \mathrm{mol} / 1$

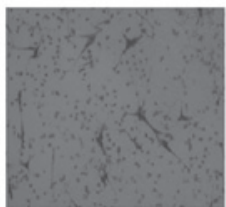

$40 \mu \mathrm{mol} / 1$

B

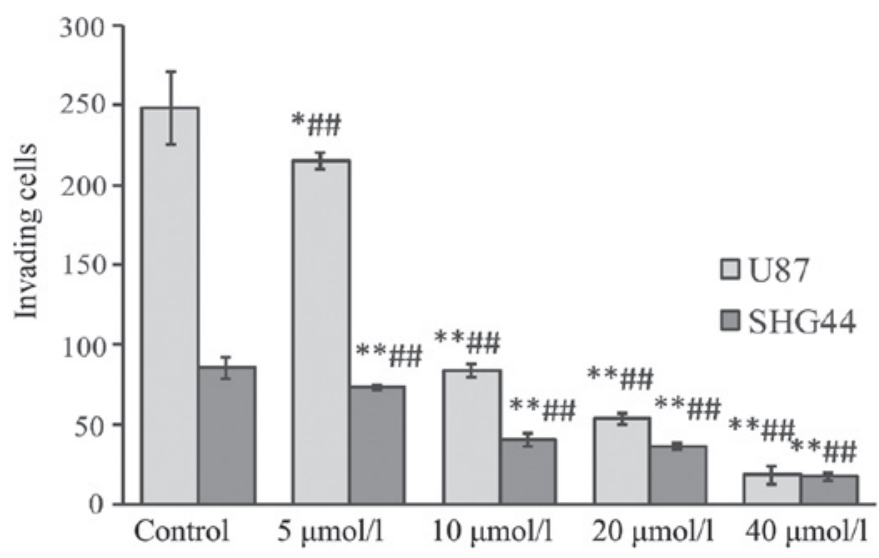

Figure 2. (A) Effect of all-trans retinoic acid (ATRA) on the invasive ability of U87 and SHG44 glioma cells (magnification, x200). (B) Number of invading glioma cells following treatment with different concentrations of ATRA for $24 \mathrm{~h}$. The number of invading glioma cells was significantly decreased with the increase in ATRA concentration. ${ }^{*} \mathrm{P}<0.05$ vs. control group; ${ }^{* *} \mathrm{P}<0.01$ vs. control group; ${ }^{\# \#} \mathrm{P}<0.01$ vs. any other treatment group $(\mathrm{n}=3)$.

A

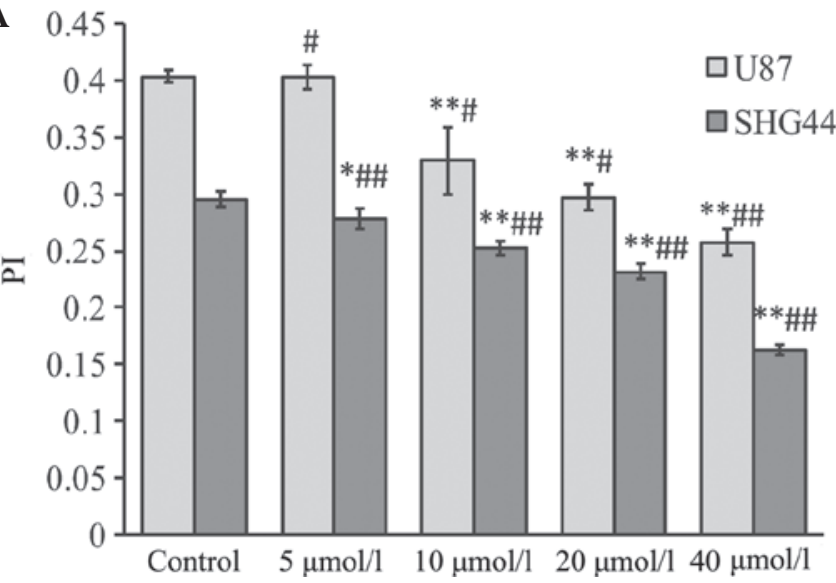

B

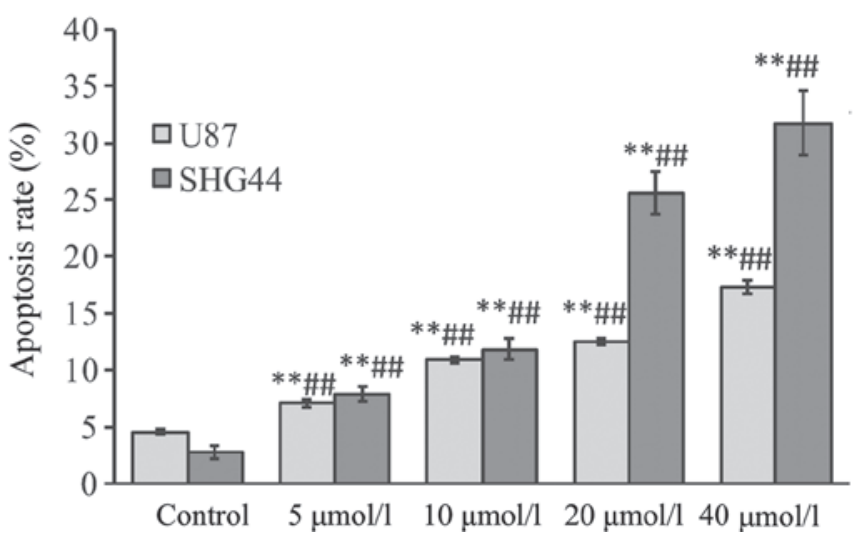

Figure 3. Effect of ATRA on the proliferation and apoptosis of glioma cells. (A) Effect of ATRA on the proliferation of glioma cells. Following treatment with different concentrations of ATRA for $24 \mathrm{~h}$, the PI of all glioma cell groups was significantly decreased, excluding in the U87 group treated with $5 \mu$ mol/1 ATRA. Furthermore, the PI decreased with the increase in ATRA concentration. (B) Effect of ATRA on the proliferation of glioma cells. Following treatment with various concentrations of ATRA for $24 \mathrm{~h}$, the apoptosis rate of the glioma cells in each treatment group was significantly increased. "P $<0.05$ vs. control group; ${ }^{* *} \mathrm{P}<0.01$ vs. control group; ${ }^{\#} \mathrm{P}<0.05$ vs. any other treatment group; ${ }^{\# \#} \mathrm{P}<0.01$ vs. any other treatment group (n=3). ATRA, all-trans retinoic acid; PI, proliferation index.

cells ( $\mathrm{P}>0.05$ vs. control group). However, following treatment with 20 or $40 \mu \mathrm{mol} / 1$ ATRA, the MMP-2 mRNA expression levels were significantly downregulated $(\mathrm{P}<0.01$ vs. control group). By contrast, treating SHG44 glioma cells with various concentrations of ATRA for $24 \mathrm{~h}$ resulted in a significant downregulation of MMP-2 mRNA expression levels at all ATRA concentrations ( $\mathrm{P}<0.01$ vs. control group; Fig. 4A).
Following treatment with various concentrations of ATRA, the variations in MMP-9 mRNA expression in the glioma cell lines were similar to those of MMP-2. The MMP-9 mRNA expression was significantly downregulated in glioma cells following treatment with different concentrations of ATRA, compared with the control group ( $\mathrm{P}<0.01$; Fig. 4B). 
A

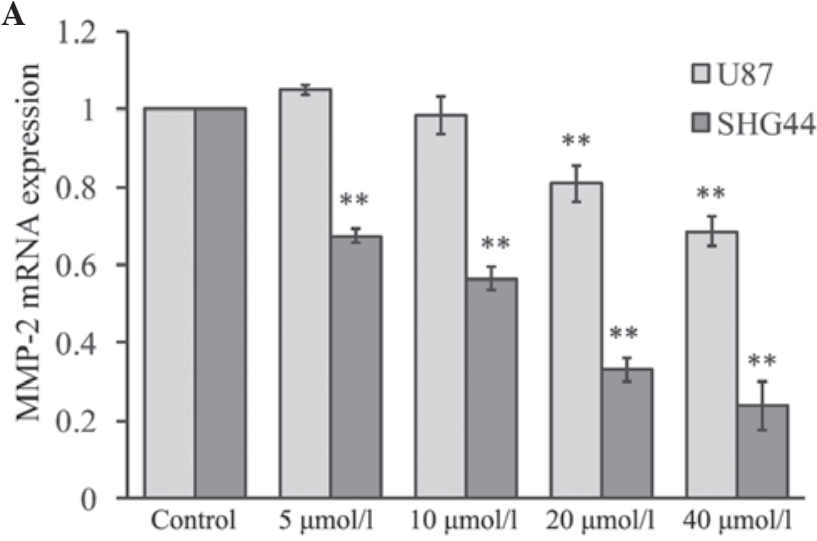

C

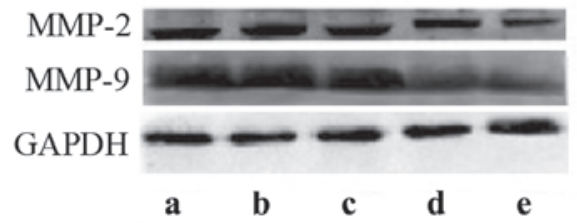

B

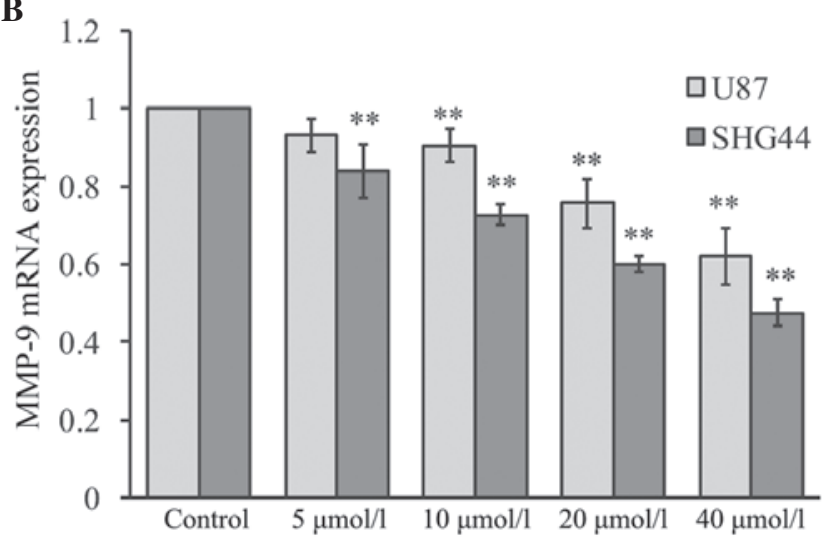

D

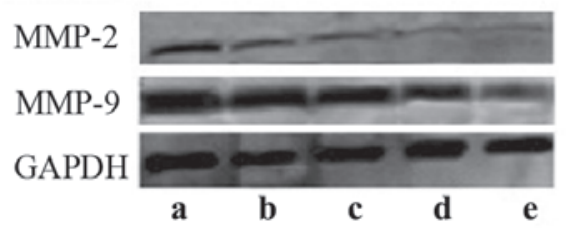

Figure 4. Effect of all-trans retinoic acid (ATRA) on the expression of MMP-2 and MMP-9 in glioma cells. Effect of ATRA on the mRNA expression levels of (A) MMP-2 and (B) MMP-9 in glioma cells. ${ }^{* *} \mathrm{P}<0.01$ vs. control group (n=3). Effect of ATRA on the protein expression levels of MMP-2 and MMP-9 in (C) U87 and (D) SHG44 glioma cells. a, control group; b, $5 \mu \mathrm{mol} / 1$; c, $10 \mu \mathrm{mol} / 1$; d, $20 \mu \mathrm{mol} / 1$; e, $40 \mu \mathrm{mol} / 1$. MMP, matrix metalloproteinases.

MMP protein in each group was examined by western blotting, as indicated in Fig. 4C and D. Changes in MMP-2 and MMP-9 protein expression levels exhibited a similar trend to the changes in MMP-2 and MMP-9 mRNA expression levels. Thus, high concentrations of ATRA may significantly downregulate the protein expression levels of MMP-2 and MMP-9 in each glioma cell line.

\section{Discussion}

Numerous studies have reported that ATRA can inhibit the migration and invasion of various types of tumor cell lines, such as human breast cancer cells (15), gastric cancer cells (16), thyroid cancer cells (17) and colon cancer cells (18). However, the effects of ATRA on the migration and invasiveness of glioma remain poorly understood. In the present study, it was identified that ATRA could significantly inhibit the migration and invasiveness of glioma cell lines in a dose-dependent manner. As gelatinases are important in the migration and invasiveness, the effects of ATRA treatment on the expression of MMP-2 and MMP-9 gelatinases were examined in two glioma cell lines. Following treatment with ATRA, MMP-2 expression was inconsistent between the various glioma cell lines. ATRA appeared to significantly inhibit MMP-2 expression in the SHG44 glioma cell line in a dose-dependent manner. By contrast, only high concentrations of ATRA inhibited MMP-2 expression in the U87 glioma cell line. Furthermore, MMP-9 expression was significantly decreased following treatment with ATRA in the two glioma cell lines. However, there was no statistically significant difference between the 5 and $10 \mu \mathrm{mol} / 1$ group of U87 glioma cells. The mechanisms that resulted in the various gelatinase expression levels induced by ATRA in the two glioma cell lines remains unclear. According to existing evidence, this may be partially associated with variations in the distribution of RAR isoforms between cell lines (19).

As ATRA inhibits the expression of gelatinases in glioma cells, it may be proposed that the inhibitory effects of ATRA on the migration and invasion of glioma cells may partially depend on the inhibitory effects of ATRA on the expression of MMP-2 and MMP-9. However, the present study identified that the expression of MMP-2 and MMP-9 in the U87 glioma cell line was not completely compatible with the variation in invasion and migration ability following treatment with various concentrations of ATRA. These results indicate that ATRA may also regulate the migration and invasion of glioma cells using alternate mechanisms. Previous studies have reported that ATRA can inhibit the activity of MMP-2 (20) and MMP-9 (15) via tissue inhibitor of metalloproteinase, a protein that may be involved in the regulation of migration and invasion of ATRA-treated glioma cells.

Although it is universally accepted that ATRA can induce apoptosis and inhibit proliferation in glioma cells $(4,20,21)$, the association between the concentration of ATRA and its effects remain unclear. In the present study, ATRA treatment significantly inhibited the proliferation and increased the apoptosis of glioma cells in a dose-dependent manner. Previous studies reported similar results in melanoma cells treated with various concentrations of ATRA (22). The mechanisms by which ATRA regulates the apoptosis of glioma cells may be associated with the caspase-3/poly(ADP-ribose) polymerase-1 signaling pathway (23-25). In addition, the downregulation of B cell lymphoma-2 (Bcl-2) and the upregulation of Bcl-2-associated $X$ protein may be involved in this process $(4,20,24)$. Furthermore, it has been reported that ATRA can inhibit the expression of cyclin D1 and c-myc, proteins that are important in the regulation of the cell cycle and can inhibit cellular proliferation (6). 
In conclusion, the present study identified that ATRA treatment may inhibit the migration and invasion of glioma cells in a dose-dependent manner, and these effects may be partially associated with the effect of ATRA on the expression of gelatinases. Furthermore, ATRA may inhibit proliferation and increase apoptosis in glioma cells. Thus, the results of the present study reveal additional information regarding the anti-glioma mechanisms of ATRA.

\section{References}

1. Nishikawa R: Standard therapy for glioblastoma - a review of where we are. Neurol Med Chir (Tokyo) 50: 713-719, 2010.

2. Clarke J, Butowski N and Chang S: Recent advances in therapy for glioblastoma. Arch Neurol 67: 279-283, 2010.

3. Stupp R, Mason WP, van den Bent MJ, et al; European Organisation for Research and Treatment of Cancer Brain Tumor and Radiotherapy Groups; National Cancer Institute of Canada Clinical Trials Group: Radiotherapy plus concomitant and adjuvant temozolomide for glioblastoma. N Engl J Med 352: 987-996, 2005

4. Zang C, Wächter M, Liu H, et al: Ligands for PPARgamma and RAR cause induction of growth inhibition and apoptosis in human glioblastomas. J Neuroncol 65: 107-118, 2003.

5. Tang K, Cao L, Fan SQ, et al: Effect of all-trans-retinoic acid on C6 glioma cell proliferation and differentiation. Zhong Nan Da Xue Xue Bao Yi Xue Ban 33: 892-897, 2008.

6. Chang Q, Chen Z, You J, et al: All-trans-retinoic acid induces cell growth arrest in a human medulloblastoma cell line. J Neurooncol 84: 263-267, 2007.

7. Li S, Gao Y, Pu K, Ma L, Song X and Liu Y: All-trans retinoic acid enhances bystander effect of suicide-gene therapy against medulloblastomas. Neurosci Lett 503: 115-119, 2011.

8. Das A, Banik NL and Ray SK: Molecular mechanisms of the combination of retinoid and interferon-gamma for inducing differentiation and increasing apoptosis in human glioblastoma T98G and U87MG cells. Neurochem Res 34: 87-101, 2009.

9. Haque A, Banik NL and Ray SK: Emerging role of combination of all-trans retinoic acid and interferon-gamma as chemoimmunotherapy in the management of human glioblastoma. Neurochem Res 32: 2203-2209, 2007.

10. Zhang R, Banik NL and Ray SK: Combination of all-trans retinoic acid and interferon-gamma upregulated p27(kip1) and down regulated CDK2 to cause cell cycle arrest leading to differentiation and apoptosis in human glioblastoma LN18 (PTEN-proficient) and U87MG (PTEN-deficient) cells. Cancer Chemother Pharmacol 62: 407-416, 2008.

11. Karmakar S, Banik NL, Patel SJ and Ray SK: Combination of all-trans retinoic acid and taxol regressed glioblastoma T98G xenografts in nude mice. Apoptosis 12: 2077-2087, 2007.
12. Karmakar S, Banik NL and Ray SK: Combination of all-trans retinoic acid and paclitaxel-induced differentiation and apoptosis in human glioblastoma U87MG xenografts in nude mice. Cancer 112: 596-607, 2008.

13. Onishi M, Ichikawa T, Kurozumi K and Date I: Angiogenesis and invasion in glioma. Brain Tumor Pathol 28: 13-24, 2011.

14. Livak KJ and Schmittgen TD: Analysis of relative gene expression data using real-time quantitative PCR and the 2(-Delta Delta C(T)) method. Methods 25: 402-408, 2001

15. Dutta A, Sen T and Chatterjee A: All-trans retinoic acid (ATRA) downregulates MMP-9 by modulating its regulatory molecules. Cell Adhes Migr 4: 409-418, 2010.

16. Yang P, Liu Z, Wang H, et al: Enhanced activity of very low density lipoprotein receptor II promotes SGC7901 cell proliferation and migration. Life Sci 84: 402-408, 2009.

17. Lan L, Cui D, Luo Y,Shi BY, Deng LL, Zhang GY and Wang H: Inhibitory effects of retinoic acid on invasiveness of human thyroid carcinoma cell lines in vitro. J Endocrinol Invest 32: 731-738, 2009.

18. Adachi Y, Itoh F, Yamamoto H, Iku S, Matsuno K, Arimura Y and Imai K: Retinoic acids reduce matrilysin (matrix metalloproteinase 7) and inhibit tumor cell invasion in human colon cancer. Tumour Biol 22: 247-253, 2001.

19. Zhou TB and Qin YH: The potential mechanism for the different expressions of gelatinases induced by all-trans retinoic acid in different cells. J Recept Signal Transduct Res 32: 129-133, 2012.

20. Papi A, Bartolini G, Ammar K, et al: Inhibitory effects of retinoic acid and IIF on growth, migration and invasiveness in the U87MG human glioblastoma cell line. Oncol Rep 18: 1015-1021, 2007.

21. Haque A, Das A, Hajiaghamohseni LM, Younger A, Banik NL, and Ray SK: Induction of apoptosis and immune response by all-trans retinoic acid plus interferon-gamma in human malignant glioblastoma T98 G and U87MG cells. Cancer Immunol Immunother 56: 615-625, 2007.

22. Zhang H, Satyamoorthy K, Herlyn M and Rosdahl I: All-trans retinoic acid (atRA) differentially induces apoptosis in matched primary and metastatic melanoma cells - a speculation on damage effect of atRA via mitochondrial dysfunction and cell cycle redistribution. Carcinogenesis 24: 185-191, 2003.

23. Gumireddy K, Sutton LN, Phillips PC and Reddy CD: All-trans-retinoic acid-induced apoptosis in human medulloblastoma: activation of caspase-3/poly (ADP-ribose) polymerase 1 pathway. Clin Cancer Res 9: 4052-4059, 2003.

24. Ran L, Tan W, Tan S, Zhang R, Wang W and Zeng W: Effects of ATRA, acitretin and tazarotene on growth and apoptosis of Tca8113 cells. J Huazhong Univ Sci Technolog Med Sci 25: 393-396, 2005.

25. Yu Z, Han J, Lin J, Xiao Y, Zhang X and Li Y: Apoptosis induced by atRA in MEPM cells is mediated through activation of caspase and RAR. Toxicol Sci 89: 504-509, 2006. 